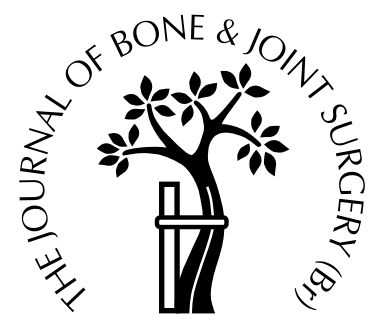

\title{
Roentgen single-plane photogrammetric analysis (RSPA)
}

\section{A NEW APPROACH TO THE STUDY OF MUSCULOSKELETAL MOVEMENT}

\author{
X. Yuan, L. Ryd, K. E. Tanner, L. Lidgren \\ From Lund and Linköping University Hospitals, Sweden
}

W e present a new approach for the accurate reconstruction of three-dimensional skeletal positions using roentgen single-plane photogrammetric analysis (RSPA). This technique uses a minimum of three markers embedded in each segment which allow continuous, real-time, internal skeletal movement to be measured from single-plane images, provided that the precise distance between the markers is known.

A simulation study indicated that the error propagation in this approach is influenced by focus position, object position, the number of control points, the accuracy of the previous measurement of the distance between markers and the accuracy of image measurement. For reconstruction of normal movement of the knee with an input measurement error of SD = $0.02 \mathrm{~mm}$, the rotational and translational differences between reconstructed and original movement were less than $0.27^{\circ}$ and $0.9 \mathrm{~mm}$, respectively.

Our results showed that the accuracy of RSPA is sufficient for the analysis of most movement of joints. This approach can be applied in combination with force measurements for dynamic studies of the musculoskeletal system.

J Bone Joint Surg [Br] 2002;84-B:908-14

Received 14 March 2000; Accepted after revision 15 October 2001

The analysis of real-time musculoskeletal movement during functional activities is of particular importance for a variety of orthopaedic investigations. The skeleton can be mod-

X. Yuan, Research Associate

Imaging Laboratory, The John P. Robarts Research Institute, London, Ontario, Canada.

L. Ryd, MD, PhD, Associate Professor, Head

Department of Orthopaedics, University Hospital, S-581 85 Linköping, Sweden.

K. E. Tanner, D Phil, Professor of Biomedical Materials Queen Mary \& Westfield College, University of London, London, UK.

L. Lidgren, Professor and Chairman

Department of Orthopaedics, University Hospital, S-221 85 Lund, Sweden.

Correspondence should be sent to Dr L. Ryd.

(C)2002 British Editorial Society of Bone and Joint Surgery 0301-620X/02/611146\$2.00 elled as a series of rigid bodies with its movement described by rigid body motion. ${ }^{1,2}$ There are many quantitative techniques for analysing this movement, either in three dimensions with six degrees of freedom (dof), ${ }^{3,4}$ or in two dimensions with three dof. ${ }^{5}$ Since the geometry of skeletal components is complex, three-dimensional analysis reveals more of skeletal movement than two-dimensional assessment.

The key issue in the analysis of skeletal movements is positional reconstruction. Traditionally, there have been two approaches for reconstruction of the three-dimensional (3D) position of an object: displacement transducers ${ }^{6}$ and RSA. ${ }^{2,7}$ Displacement transducers are particularly difficult to use in many clinical situations, especially for internal movements, while the use of RSA is more convenient and has become popular. ${ }^{1,2,8-10}$ Usually, RSA requires markers to represent skeletal orientations and positions. These can be either attached externally to the surface of skeletal segments or injected internally into bone. External markers can be traced continuously, allowing real-time measurement, ${ }^{11}$ but because of the relative movement between the skeleton and overlying soft tissue or skin, they are limited in their representation of skeletal positions. By contrast, internal markers reveal real skeletal positions by radiography, but because of exposure limits in conventional radiographic systems, most of these measurements are confined to single-time measurement. Recently, digital radiographic systems, such as flat-panel digital radiography $^{12}$ and digital fluoroscopy, ${ }^{13}$ have allowed skeletal positions to be traced continuously. Theoretically, the use of two radiographic sources to establish an RSA system could provide an efficient method for detecting continuous skeletal movements. Clinically, however, this method limits the available space for skeletal movement and is expensive and not widely available.

Consequently, the possibility of performing reconstruction using three-dimensional marker co-ordinates from a single-plane view has been explored. The stereo approach has received much attention whereas singleplane photogrammetry has not. Recently, a new method of analysis of movement for the implanted knee with a single-plane image has been studied. ${ }^{14-16}$ In this approach, a library of implanted prosthetic geometrics, which contains all six dof images, has to be created in 


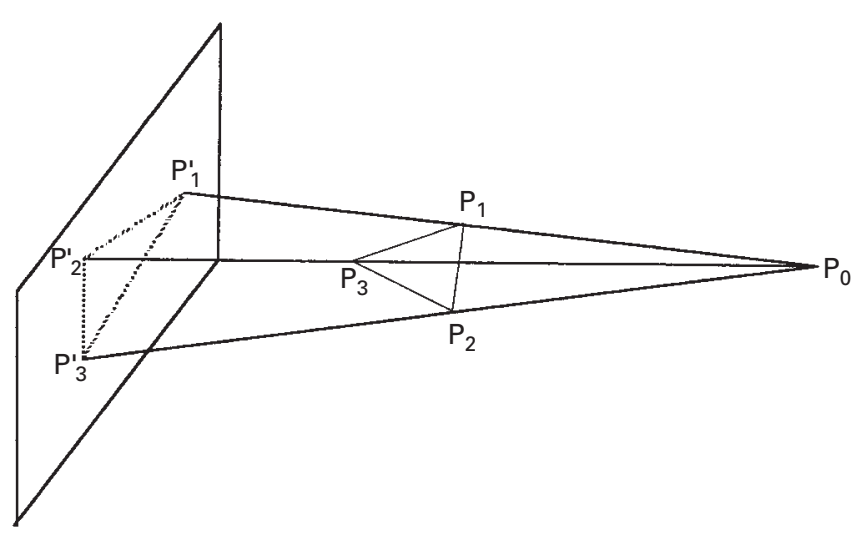

Fig. 1

Diagram showing the outline of reconstruction of 3D object point positions. $\mathrm{P}_{0}$ is the point of projective centre, $\mathrm{P}_{\mathrm{i}}^{\prime}(\mathrm{i}=1$ to $\mathrm{n}$ ) are projective points on image plane and $\mathrm{P}_{\mathrm{i}}(\mathrm{i}=1$ to $\mathrm{n})$ are object points.

advance and the in vivo single-plane prosthetic geometrical pattern is then matched with that in the library, enabling the position of the prosthesis and thus the movement to be estimated. Since this approach requires geometrical information for different types and sizes of prosthesis, it has limited practical application.

We describe a technique for reconstructing the threedimensional marker co-ordinates based on a single-plane RSA method, which we have called RSPA. The problem can be stated as follows. Given a cinematic sequence of $\mathrm{x}$ ray images of a skeletal subject with several markers and accurately knowing the distances between the markers, how can the three-dimensional positions of the markers be determined? The error propagation of RSPA was studied by computer simulation. RSPA differs from the other methods in that real-time, continuous accurate three-dimensional skeletal movement can be detected using only single-plane images provided that the distances between the markers are known. Combined with a force measurement system, this method could, in the future, be applied to the study of the dynamics of the musculoskeletal system.

\section{Materials and Methods}

A simplification of the proposed RSPA model is shown in Figure 1 and the detailed mathematical background, the derivation of the formula and two methods for solving the equations are given in the Appendix. In this study, method 1 was used, for reasons of simplicity.

Apparatus for measurement. One precondition to obtain solutions to equation A5 (see Appendix) is knowledge of the projective centre $\mathrm{P}_{0}$, or focus position. In our study, this position was found using a calibration cage with two functional planes called the fiducial and control planes. The function of the fiducial plane was to establish a transformation relationship between the image measurement co-ordinate system and the cage coordinate system, while that of the control plane was to reconstruct the focus position. The mathematical descriptions which were employed to determine transformation and focus position using the calibration cage were similar to those for conventional RSA.,17

We designed a calibration cage (Fig. 2) in which the fiducial and control planes were both square with dimensions of $220 \pm 220 \mathrm{~mm}^{2}$ and parallel $250 \mathrm{~mm}$ apart. The fiducial plane contained 40 tantalum beads, called fiducial marks (FM), embedded every $20 \mathrm{~mm}$ around the periphery of the fiducial plane. The control plane contained 321 titanium beads, named control points (CP), $10 \mathrm{~mm}$ or $5 \mathrm{~mm}$ apart at locations near the centre of the plane. Both the FM and $\mathrm{CP}$ were distributed so that normally their images did not overlap. A 3D cage co-ordinate system, xyz, was defined so that the origin of the co-ordinate system was located at the centre of the fiducial plane. The $\mathrm{x}$ - and $\mathrm{y}$-axes were in the fiducial plane with the $\mathrm{x}$-axis horizontal and the

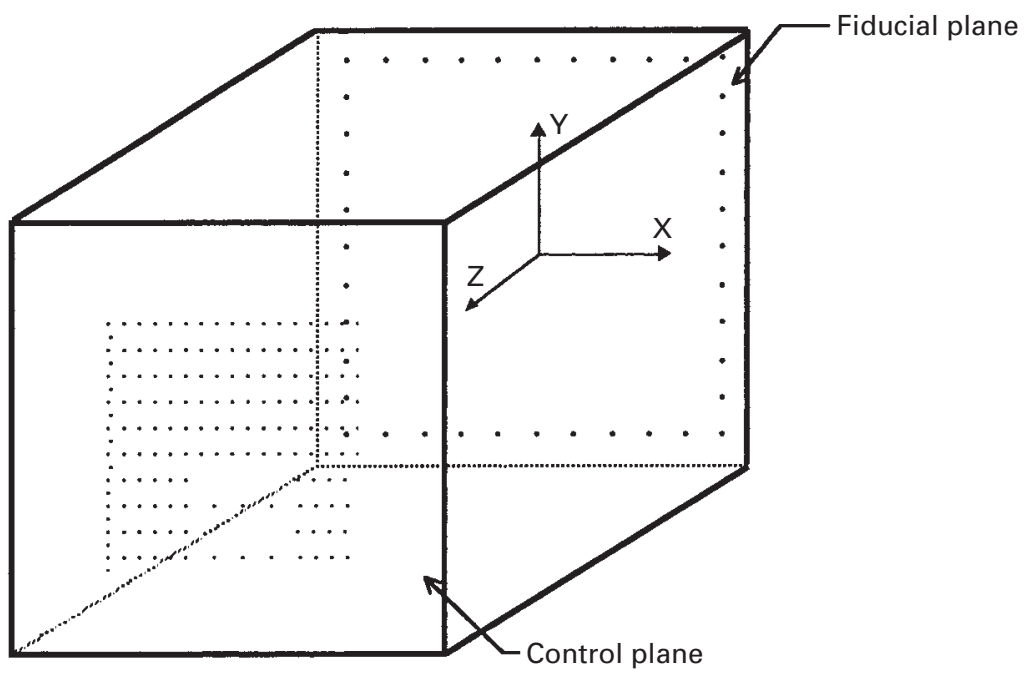

Fig. 2

Diagram showing the calibration cage of RSPA with 40 FM and $321 \mathrm{CP}$. 


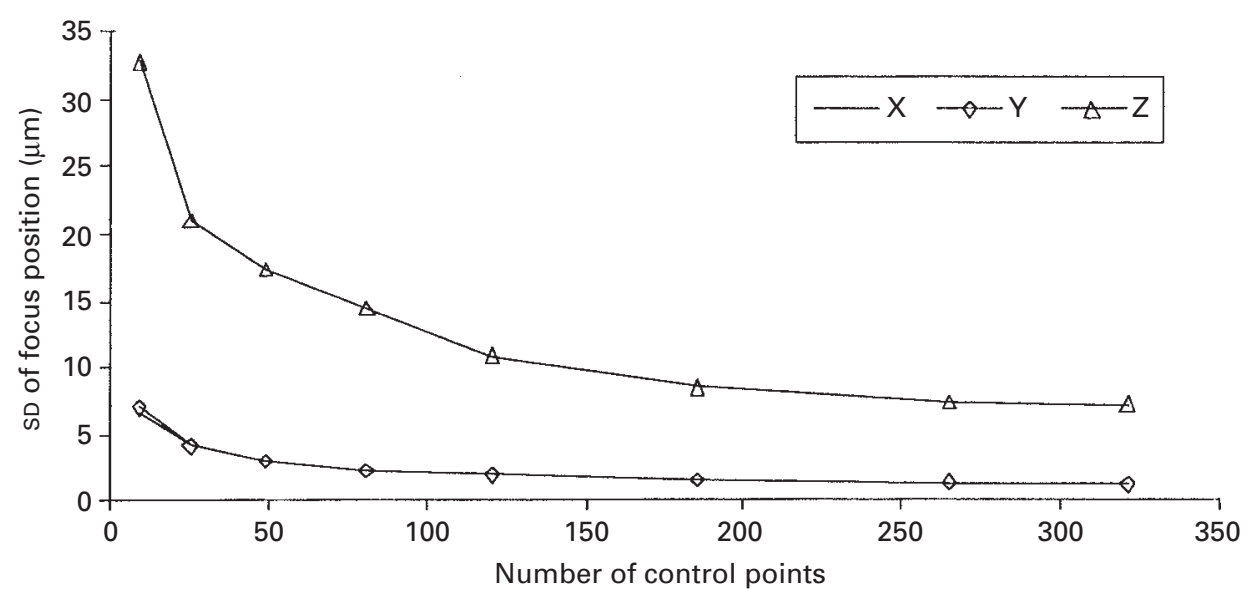

Fig. 3a

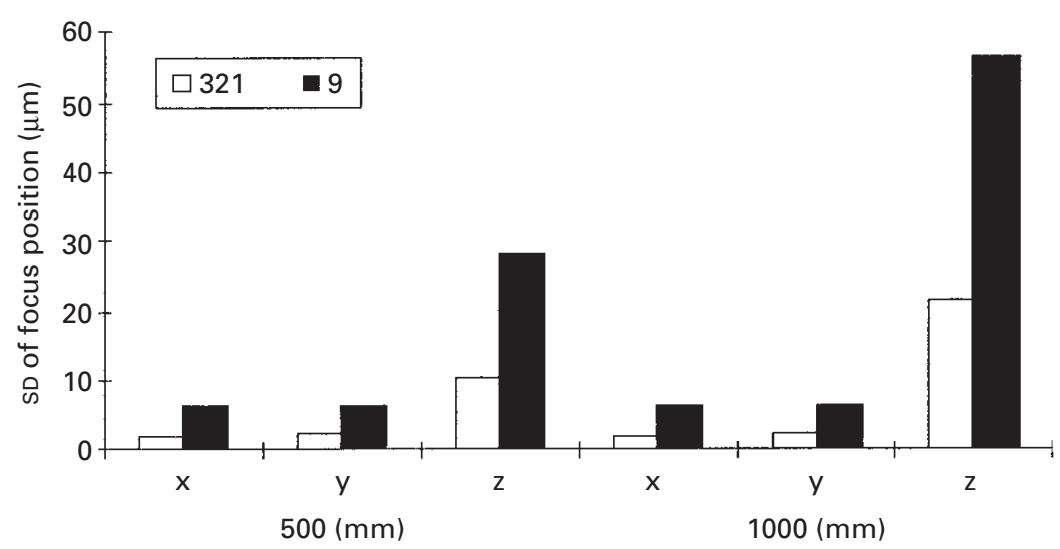

Fig. 3b

Figure 3a - Graph showing the influence of CP number on the accuracy of reconstructed focus position. Accuracies for eight sets of CP numbers: 9, 25, 49, 81, 121, 185, 265 and 321 were calculated with input errors of $\mathrm{SD}=0.02 \mathrm{~mm}$. Figure $3 \mathrm{~b}-$ Graph showing the influence of CP number and focus position on the accuracy of reconstructed focus position in RSPA. The errors are for two sets of CPs containing 9 and 321 points and two distances from focus position to the fiducial plane, 500 and $1000 \mathrm{~mm}$ with the input errors of $\mathrm{SD}=0.02 \mathrm{~mm}$.

$\mathrm{y}$-axis vertical. The $\mathrm{z}$-axis is perpendicular to the $\mathrm{x}-\mathrm{y}$ plane following the criteria of right-hand orientation.

Technique. The practical procedures to perform RSPA are as follows: 1) to determine the distance between the skeletal markers, called object points (OP), which can be done by direct measurements if the markers are attached to the implanted components of an endoprosthesis, or by conventional RSA measurement if the markers are injected into the bone; 2) to calibrate the system by obtaining a calibration cage image and then removing the cage; 3 ) to obtain consecutive subject movement images; 4) to measure the images to obtain all the marker co-ordinates which include the FM and $\mathrm{CP}$ on the calibration image and object points on the other images; 5) to calculate the focus position from the calibration image; 6) to reconstruct 3D co-ordinates of each object point; and 7) to calculate real-time movement according to the reconstructed object point positions.

Validation of the method and evaluation of the accur- acy. In order to evaluate the accuracy of the proposed RSPA approach, we performed computer simulations. The numerical error-free co-ordinates for focus position and different markers, such as fiducial marks (FM), control points $(\mathrm{CP})$ and object points $(\mathrm{OP})$ in different time sequences, were created in 3D space. The OPs chosen were positioned at the vertices of a cube of length $30 \mathrm{~mm}$. These markers were mathematically projected to the fiducial plane, then transferred to the image-measurement co-ordinate system, creating error-free measurement data. The practical measurement co-ordinates were simulated by adding random errors to these error-free measurement data. In our study, Gaussian distribution random errors with a mean of zero and SD of $0.02 \mathrm{~mm}$ were applied based on the consideration of the accuracy of the conventional manual measurement table, about $0.01 \mathrm{~mm}^{2}$, and a factor of operator handling. A computer program was developed using MATLAB (The Mathworks Inc, Natick, Massachusetts) 


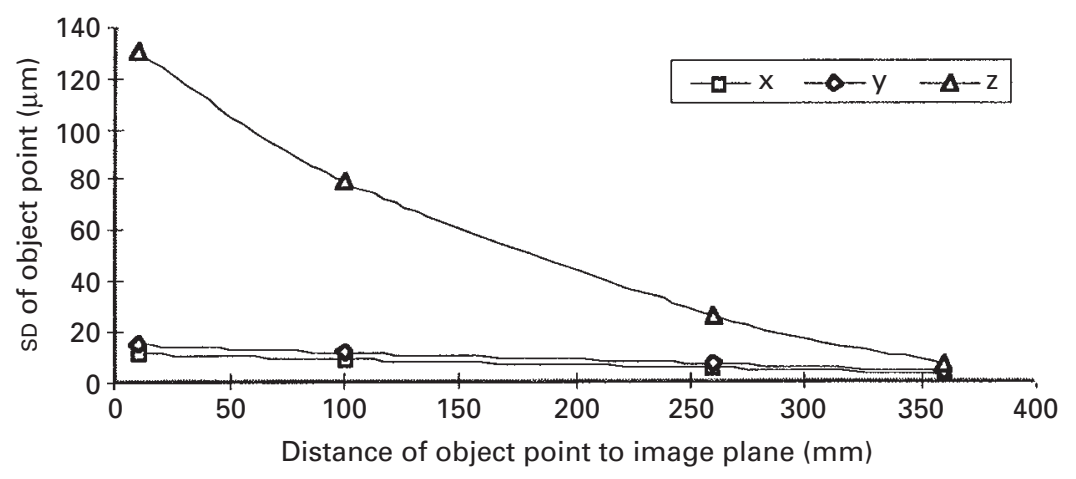

Fig. 4

Graph showing the influence of OP position in the out-of-plane dimension on the accuracy of the reconstructed co-ordinates as determined by RSPA simulation.

and the simulations were repeated 1000 times with the errors added differently each time.

Three types of simulation were performed. First, the influence of CP number and focus position was assessed by comparing the accuracy of the reconstructed focus position. The number of CPs was varied between 9 and 321. Secondly, the accuracies of reconstructed OPs were evaluated by moving the OP between the image plane and the focus. The focus position was placed 500 and $1000 \mathrm{~mm}$ from the image plane. The control plane was located midway between the focus position and fiducial plane.

Finally, a normal human knee movement trajectory over one cycle of movement, with three rotation and three translation components measured in vivo, ${ }^{10}$ was divided into 29 time intervals. Three OPs positioned at the vertices of a cube were simulated as a segment for both femur and tibia. The OP positions at these time intervals were reconstructed and then the movement components of the whole cycle were regenerated. The influence of errors on the movement trajectory calculations was divided into three components: errors in the distance between the markers, errors in image measurement data and errors in both distance and image measurement. For all these components, the maximum difference between the regenerated and original movement trajectory was compared and the critical factors which influenced the accuracy of the regenerated movement were identified.

\section{Results}

The influence of CP number (Fig. 3a) and focus position (Fig. 3b) was assessed by comparing the accuracy of the reconstructed focus position. When the number of CPs was increased from 9 to 321, the accuracy of the focus position reconstruction increased by about $83 \%$ and $78 \%$ in the $x-y$ plane and the $\mathrm{z}$-axis, respectively, with the focus located at $500 \mathrm{~mm}$. With $150 \mathrm{CPs}$ the accuracy was increased by about $74 \%$ compared with that with 9 CPs. In addition, the accuracy of the focus position was also related to its distance from the fiducial plane. Decreasing this distance from $1000 \mathrm{~mm}$ to $500 \mathrm{~mm}$ linearly increased the accuracy in the $\mathrm{z}$-axis, but had no effect on that of $\mathrm{x}-\mathrm{y}$ plane.

Comparing the different positions of OPs, we found that the accuracy of reconstructed OP was affected by their own position (Fig. 4). Shifting the object point from $10 \mathrm{~mm}$ to $360 \mathrm{~mm}$ along the $\mathrm{z}$-axis increased the accuracy in the $\mathrm{x}-\mathrm{y}$ plane and the z-axis by about $76 \%$ and $95 \%$, respectively. The accuracy of reconstructed OP co-ordinates in the outof-plane (z-) direction was about one-tenth of that in the inplane (x-y plane), when it was located at $10 \mathrm{~mm}$, while the corresponding accuracies were only a factor of two different when the OP was at $360 \mathrm{~mm}$.

The movement cycles of the original and reconstructed knees are shown in Figure 5 and the results indicated that reconstructed movement components reproduced the original movement well. Table I lists the maximum differences between reconstructed and original movement for the errors added to the distance between markers, to the measurement data and to both. Over the whole cycle, the maximum differences between reconstructed rotation and translation and the original movements were less than $0.27^{\circ}$ and $0.9 \mathrm{~mm}$.

\section{Discussion}

We have presented a new approach, RSPA, to measure realtime, continuous skeletal movement. The mathematical solutions of the RSPA technique were derived and two methods were proposed in different marker configurations. If there are only three markers in a rigid body, method 1 and method 2 are equivalent. If there are more than three markers, method 2 uses more 'equations'. From the optimal mathematical point of view, method 2 is better than method 1. In our study, method 1 was used for reasons of simplicity. In the case of noisy or error-containing data and changing positions of the markers method 2 could be more appropriate.

The analysis of the error propagation in RSPA is critical 


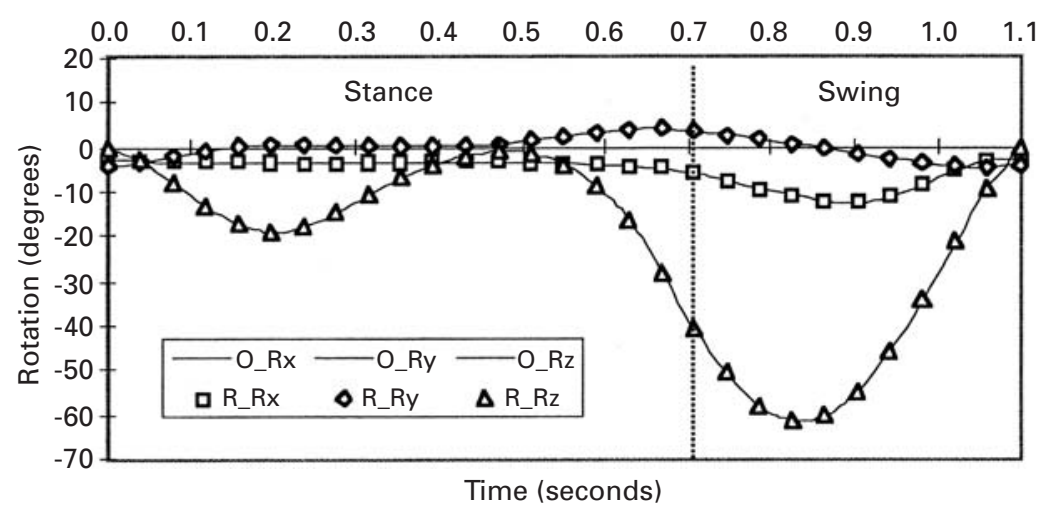

Fig. 5a

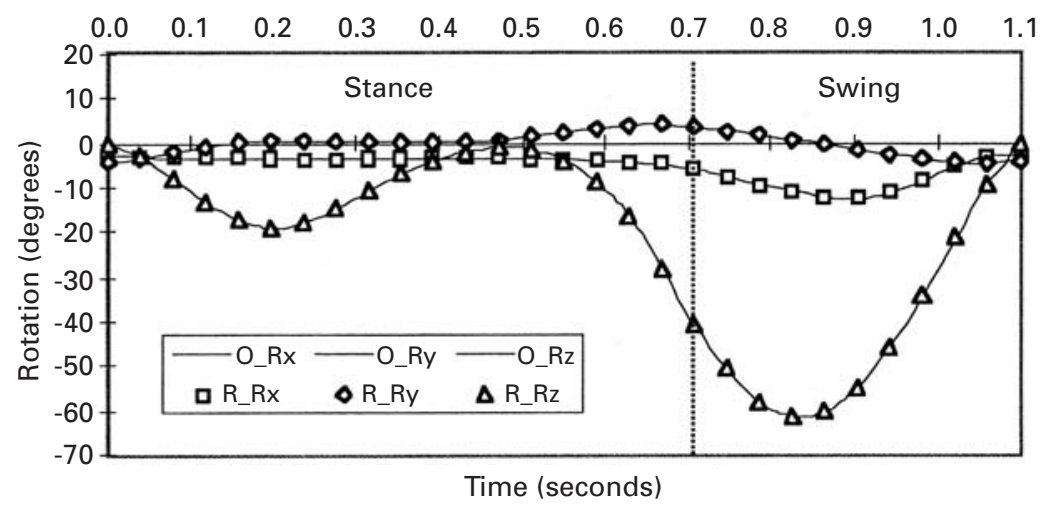

Fig. $5 b$

Figure 5a - Graph showing the rotational trajectory between original and reconstructed movement. Here Rx, Ry and Rz refer to the rotation around the $\mathrm{x}$ (adduction/abduction), $\mathrm{y}$ (internal/external) and $\mathrm{z}$ (extension/flexion) axes; and $\mathrm{O}_{-}$and $\mathrm{R}_{-}$indicate the original and reconstructed rotational trajectories with the input errors of $\mathrm{SD}=0.02 \mathrm{~mm}$. Figure $5 \mathrm{~b}-\mathrm{Graph}$ showing the comparison of translational trajectory between original and reconstructed motion. Here $\mathrm{Tx}$, Ty and $\mathrm{Tz}$ refer to the translation along the $\mathrm{x}$ (anterior/posterior), $\mathrm{y}$ (compression/distraction) and $\mathrm{z}$ (lateral/medial) axes, and $\mathrm{O}_{-}$and $\mathrm{R}_{-}$indicate the original and reconstructed translational trajectory with the input errors of $\mathrm{SD}=0.02 \mathrm{~mm}$.

Table I. The maximum difference between reconstructed and original movement. The simulated measurement error $(\mathrm{SD}=0.02 \mathrm{~mm})$ added to the $2 \mathrm{D}$ error-free measurement data, the distance between the marker and both together. See Figure 5

\begin{tabular}{lllllll}
\hline & \multicolumn{7}{l}{ Maximum difference with respect to } \\
\cline { 2 - 7 } & \multicolumn{2}{l}{ Rotation (degrees) } & \multicolumn{3}{c}{ Translation $(\mathbf{m m})$} \\
& $\Theta_{\mathbf{x}}$ & $\Theta_{\mathbf{y}}$ & $\Theta_{\mathbf{z}}$ & $\mathbf{T}_{\mathbf{x}}$ & $\mathbf{T}_{\mathbf{y}}$ & $\mathbf{T}_{\mathbf{z}}$ \\
\hline Measurement & 0.0397 & 0.0611 & 0.0297 & 0.1036 & 0.0095 & 0.1909 \\
Distance & 0.2682 & 0.2743 & 0.1317 & 0.6078 & 0.9021 & 0.2353 \\
Both above & 0.2613 & 0.2381 & 0.1442 & 0.6561 & 0.8105 & 0.1061 \\
\hline
\end{tabular}

to assess its clinical applicability. In our study, computer simulation was used to perform this analysis. Many factors, from measurement errors to distances between OPs, were examined.

The error propagation of the RSPA approach was sensitive to the focus position and the calibration cage, espe- cially the number of CPs on the cage. The distance from the focus to the image plane was approximately inversely proportional to the accuracy of the reconstructed focus in the z-axis. Thus, increasing the distance decreased the accuracy of the z-axis, but had no effect in the $x-y$ plane. Unlike the influence of the focus position, the number of CPs affected the accuracy of the reconstructed focus position in all three axes. From the geometrical projection point of view, in-plane (x-y plane) focus position error had a tangential relationship to the error of reconstructed OPs in the z-axis. Thus reducing the in-plane focus position error was important for improving OP accuracy. This phenomenon was different from conventional stereophotogrammetry in which the errors in the z-axis are controlled by the pairing image separation. Previously, ${ }^{18}$ we have found that 21 CPs were sufficient to reach high accuracy in RSA, but this number was insufficient in RSPA and 150 
CPs should be used. The formidable task of digitising 350 markers can be solved by implementing automatic systems. $^{19}$

Mathematically, two-dimensional transformation is a subset of three-dimensional transformation and error propagation in the latter has been previously investigated. ${ }^{3}$ This indicated that the accuracy of such a transformation depended on the number of markers, the error of the markers and the distance between the markers. Accordingly, we used 40 FM and set them peripherally at a minimum distance of $50 \mathrm{~mm}$ from the centre of the fiducial plane. Using the error propagation model, ${ }^{3,4}$ we estimated that rotational and translational accuracies for the transformation were $0.0063^{\circ}$ and $4.5 \mu \mathrm{m}$ respectively, when the SD of the measurement error was $0.02 \mathrm{~mm}$. The transformational accuracy was such that we assumed all the points to be well transformed.

The reconstructed OP had the lowest accuracy in the zaxis. In addition to being affected by the number of CPs and the focus position, the OP accuracy in the z-axis was also influenced by its location. The closer the OPs were to the film the worse was the accuracy. A shift towards the focus, however, reduced the measurement area which limited the object movement range. To reach the optimal OP, we needed to consider object size, its movement range and the focus position.

It should be noted that the accuracy of the input data was very important for this novel approach. Input data errors are related to the image capture equipment and marker measurement system. Reducing the marker measurement error directly improves the accuracy of RSPA. For conventional RSA measurement systems, most have a maximum accuracy of $0.01 \mathrm{~mm}$. This accuracy, however, is rarely reached in practice because of the image quality and inconsistent operator handling, etc, thus errors with an SD of $0.02 \mathrm{~mm}$ were used in this study. Recently developed automated image measurement systems provide a potential technique to reduce random errors and thus to improve the measurement accuracy. ${ }^{19-22}$ The errors incorporated by the image capture equipment, such as fluoroscopic distortion, are systematic errors and cannot be reduced by improving the measurement accuracy with an automated image measurement system. In order to reduce systematic errors, special corrections are needed, but the accuracy of such corrections is limited. ${ }^{13-15,23}$ Fortunately, a newly developed flat-panel digital $\mathrm{x}$-ray system without image distortion, ${ }^{12}$ and fluoroscopy with distortion-free digital imaging technique have been developed and may be applicable to RSPA.

Knowing the precise distance between markers is a precondition for RSPA. These distances could be measured by conventional RSA, if the markers were inserted into the bone or measured with arbitrary accuracy by a number of methods when the markers were manufactured on, for example, components of an endoprosthesis. Comparing the influence of errors in the marker distances and errors in the measurement data, we found the former had the largest influence on the reconstructed motion accuracies (Table I).
At the same level of input errors ( $\mathrm{SD}=0.02)$, the maximum differences between reconstructed and original movement generated by the errors of the marker distances were about four and nine times higher than those generated by the error in measurement data for rotation and translation, respectively. Thus in practice, obtaining high accuracy of the intermarker distances is the most important factor.

In the simulation of in vivo normal movement of the knee, the maximum difference between the original and the simulated movement for both rotation and translation was found to be $0.27^{\circ}$ and $0.9 \mathrm{~mm}$, respectively. These values are similar to the size of implanted prosthesis micromotion. RSPA could not be used therefore in situations in which high accuracy was demanded. However, its accuracy should be more than sufficient to measure movement of joints. ${ }^{24}$

We thank Dr Geneviève Dumas since part of this study was done when the first author visited her laboratory in the Department of Mechanical Engineering, Queen's University, Canada. The investigations were financially supported by Greta och Johans Kocks Stiftelse, Stiftelsen Bistånd åt Vanföra i Skåne, Konung Gustav V's 80-årsfond, NUTEK. The Medical Faculty of Lund University, the Medical Research Council project 09509 and the Swedish Foundation for Strategic Research.

No benefits in any form have been received or will be received from a commercial party related directly or indiretly to the subject of this article.

\section{APPENDIX}

The mathematical description for using single-plane photogrammetry to reconstruct $3 \mathrm{D}$ marker co-ordinates is shown in Figure 1. Considering $n$ markers $\mathbf{P}_{\mathrm{i}}\left(\mathrm{x}_{\mathrm{i}}, \mathrm{y}_{\mathrm{i}}, \mathrm{z}_{\mathrm{i}}\right)(\mathrm{i}=1 \ldots \mathrm{n})$ in space, if the position of the projective centre, that is the $\mathrm{x}$-ray focus, is $\mathbf{P}_{0}\left(\mathrm{x}_{0}, \mathrm{y}_{0}, \mathrm{z}_{0}\right)$, the marker projective coordinates are $\mathrm{Pi}^{\prime}\left(\mathrm{x}_{\mathrm{i}}^{\prime}, \mathrm{y}_{\mathrm{i}}^{\prime}, \mathrm{z}_{\mathrm{i}}^{\prime}\right)$ and the distances between different markers are $\mathrm{L}_{\mathrm{ij}}$, then we have:

$$
\begin{array}{ll}
\mathbf{P}_{i}=\mathbf{P}_{0}-\lambda_{i}\left(\mathbf{P}_{\mathrm{i}}^{\prime}-\mathbf{P}_{\mathrm{i}}^{\prime}\right) & \mathrm{i}=1 \cdots \mathrm{n} \\
\left|\mathbf{P}_{\mathrm{i}}-\mathbf{P}_{\mathrm{j}}\right|=L_{\mathrm{ij}} & \mathrm{i}=2 \cdots \mathrm{n} \\
\mathrm{j}=1 \cdots(\mathrm{i}-1)
\end{array}
$$

where $\lambda_{\mathrm{i}}$ is a proportional parameter in the range $0<\lambda_{\mathrm{i}}<$ 1. If distance vector $\mathbf{P}_{\mathrm{i}}-\mathbf{P}_{\mathrm{j}}$ is expressed by co-ordinates components from equation A1, we have:

$$
\mathbf{A}_{\mathrm{ij}}=\lambda_{\mathrm{j}} \mathbf{B}_{\mathrm{j}}-\lambda_{\mathrm{i}} \mathbf{B}_{\mathrm{i}}
$$

where

$$
\mathbf{A}_{\mathrm{ij}}=\left\{\begin{array}{l}
x_{\mathrm{i}}-x_{\mathrm{j}} \\
y_{\mathrm{i}}-y_{\mathrm{j}} \\
z_{\mathrm{i}}-z_{\mathrm{j}}
\end{array}\right\} ?, \mathbf{B}_{\mathrm{i}}=\left\{\begin{array}{ll}
x_{0}-x_{\mathrm{i}}^{\prime} \\
y_{0}-y_{\mathrm{i}}^{\prime} \\
z_{0}-z_{\mathrm{i}}^{\prime}
\end{array}\right\} ? \begin{aligned}
& \mathrm{i}=2 \cdots \mathrm{n} \\
& \mathrm{j}=1 \cdots(\mathrm{i}-1)
\end{aligned}
$$

Substituting equation A3 to equation A2, we finally derive:

$$
\lambda_{\mathrm{i}}^{2} D_{\mathrm{ii}}-2 \lambda_{\mathrm{i}} \lambda_{\mathrm{j}} D_{\mathrm{ij}}+\lambda_{\mathrm{j}}^{2} D_{\mathrm{jj}}=L_{\mathrm{ij}}^{2} \quad \begin{aligned}
& \mathrm{i}=2 \cdots \mathrm{n} \\
& \mathrm{j}=1 \cdots(\mathrm{i}-1)
\end{aligned}
$$


where $D_{i j}$ is equal to:

$$
D_{\mathrm{ij}}=\mathbf{B}_{\mathrm{i}}^{\mathrm{T}} \cdot \mathbf{B}_{\mathrm{j}} \quad \begin{aligned}
& \mathrm{i}=2 \cdots \mathrm{n} \\
& \mathrm{j}=1 \cdots(\mathrm{i}-1)
\end{aligned}
$$

which relates to the position of projective centre $\mathbf{P}_{0}$ and projective co-ordinates $\mathbf{P}_{\mathrm{i}}^{\prime}$ and $\mathbf{P}_{\mathrm{j}}$.

Equation A5 defines a series of non-linear, multivariable equations which express multiple surfaces in space and the solutions of these equations occur at the intersection points of these surfaces. If there are less than three markers, there are infinite solutions for variables $\lambda_{i}(i=1 \ldots n)$, so that marker positions $\mathrm{P}_{\mathrm{i}}$ cannot be determined. However, if the number of markers is equal to three then a single solution exists and if more than three markers are used, equation A5 becomes a set of overdetermined equations and finite solutions for variable $\lambda_{\mathrm{i}}(\mathrm{i}=1 \ldots \mathrm{n})$ can be obtained.

Generally, there is no prescription for finding the solutions. To analyse the problem, we considered two methods of searching for the solutions.

Method 1. When the number of markers is equal to or greater than three, we considered the three most widely separated markers and rewrote the corresponding three equations to express one variable as a function of the other two. The remaining two equations could then be written as functions of two variables. Two simultaneous equations were solved to obtain solutions for these two variables. Substituting the solutions obtained back to the remaining equations, the solutions for the other variables were obtained. Normally, there are eight solutions for each variable, but only two are positive, real solutions within the range of zero to one $(0,1)$. The correct solution of these two can be identified by follow-up calculation of movement.

Method 2. Practically, when the number of markers is greater than three, the solutions of variables $\lambda_{i}(i=1 \ldots n)$ can be obtained by minimising the following cost functions $\mathbf{F}_{\mathrm{ij}}$ under the non-linear least-squares sense:

$$
\begin{aligned}
& \mathbf{F}_{\mathrm{ij}}\left(\lambda_{\mathrm{i}}, \lambda_{\mathrm{j}}\right)=\lambda_{\mathrm{i}}^{2} D_{\mathrm{ii}}-2 \lambda_{\mathrm{i}} \lambda_{\mathrm{j}} D_{\mathrm{ij}}+\lambda_{\mathrm{j}}^{2} D_{\mathrm{jj}}-\mathrm{L}_{\mathrm{ij}}^{2} \quad \mathrm{i}=2 \cdots \mathrm{n} \\
& \mathrm{j}=1 \cdots(\mathrm{i}-1) \\
& \text { n } i-1 \\
& \min _{\lambda(\mathrm{a}, \mathrm{b}) \mathrm{i}=2 \mathrm{j}=1} \sum_{\mathrm{j}=1}^{i-1}\left(\mathbf{F}_{\mathrm{ij}}\left(\lambda_{\mathrm{i}}, \lambda_{\mathrm{j}}\right)\right)^{2}
\end{aligned}
$$

If physical considerations suggest a solution to the problem should lie near point $\lambda_{i}^{0}$, the search for the solution to equation A7 can often be accelerated greatly by selecting $\lambda_{i}$ as the initial solution. In the study of continuous realtime movement, it is assumed that the position of each marker changes only slightly in sequential image frames. Thus the solutions of variables for the previous image frame can be taken as the initial values $\lambda_{i}^{0}$ for the sequence image frame and a search range $(a, b)$ can be set near the initial values by taking $(a, b)=\lambda_{i}^{0} \pm \epsilon_{i}$ where the values of $\epsilon_{\mathrm{i}}$ may be defined depending on the motion under consideration.
Once the solutions for $\lambda_{\mathrm{i}}$ were determined, they are substituted to equation A1 to obtain the $3 \mathrm{D}$ co-ordinates of each marker.

\section{References}

1. Ryd L. Micromotion in knee arthroplasty: a roentgen stereophotogrammetric analysis of tibial component fixation. Acta Orthop Scand 1986;57:Suppl 220.

2. Selvik G. Roentgen stereophotogrammetry: a method for the study of the kinematics of the skeletal system. Acta Orthop Scand 1989;232Suppl:1-51.

3. Woltring HJ, Huiskes R, de Lange A, Veldpaus FE. Finite centroid and helical axis estimation from noisy landmark measurements in the study of human joint kinematics. J Biomech 1985;18:379-89.

4. Yuan X, Ryd L, Blankevoort L. Error propagation for relative motion determined from marker positions. J Biomech 1997;30:989-92.

5. Stiehl JB, Komistek RD, Dennis DA, Paxon RD, Hoff WA. Fluoroscopic analysis of kinematics after posterior-cruciate-retaining knee arthroplasty. J Bone Joint Surg [Br] 1995;77-B:884-9.

6. Gilbert JL, Bloomfeld RS, Lautenschlager EP, Wixson RL. A computer-based biomechanical analysis of the three-dimensional motion of cementless hip prostheses. J Biomech 1992;25:329-40.

7. Lee S, Harris KG, Nassif J, Goel VK, Clark CR. In vivo kinematics of the cervical spine. Part I: development of a roentgen stereophotogrammetric technique using metallic markers and assessment of its accuracy.J Spinal Disord 1993;6:522-34.

8. Koh TJ, Grabiner MD, De Swart RJ. In vivo tracking of the human patella. J Biomech 1992;25:637-43.

9. Kärrholm J. Roentgen stereophotogrammetry: review of orthopaedic applications. Acta Orthop Scand 1989;60:491-503.

10. Lafortune MA, Cavanagh PR, Sommer HJ, Kalenak A. Three dimensional kinematics of the human knee during walking. J Biomech 1992;25:347-57.

11. Morrison JB. The forces transmitted by the human knee joint during activity. PhD Thesis, University of Strathclyde, 1967.

12. Yaffe MJ, Rowlands JA. X-ray detectors for digital radiography. Phys Med Biol 1997;42:1-39.

13. Flynn M, Tashman S, Cverna F, Dupre K, Reimann D. Evaluation of high speed joint kinematics with a biplane digital radiographic system. In: Spie, ed. Medical imaging symposium Newport Beach, USA, 1996.

14. Banks SA, Hodge WA. Accurate measurement of three-dimensional knee replacement kinematics using single-plane fluoroscopy. IEEE Trans Biomed Eng 1996;43:638-49.

15. Hoff WA, Komistek RD, Dennis DA, Gabriel SM, Walker SA. Three-dimensional determination of femoral-tibial contact positions under in vivo conditions using fluoroscopy. Clin Biomech (Bristol, Avon) 1998;13:455-72.

16. Mawatari T, Miura $\mathbf{H}$, Higaki $\mathbf{H}$, et al. Pattern matching method for the 3-D kinematic analysis of total knee arthroplasty in vivo. In: Combined ORS Jaan: Hamamastsu, 1998:101.

17. Söderkvist I, Wedin PA. Determining the movements of the skeleton using well-configured markers. J Biomech 1993;26:1473-7.

18. Yuan X, Ryd L. Accuracy analysis for RSA: a computer simulation study on 3D marker reconstruction.J Biomech 2000;33:493-8.

19. Vrooman HA, Valstar ER, Brand GJ, et al. Fast and accurate automated measurements in digitized stereophotogrammetric radiographs. J Biomech 1998;31:491-8.

20. Börlin N, Kärrholm J. Radiostereometry based on digitised radiographs. In: 43rd Annual Trans ORS Meeting. 1997.

21. Gill HS, Alfaro J, Murray DW, Marks B. A new hybrid video/ digitiser based roentgen stereometric measurements system. J Biomech 1998;31:88.

22. Østgaard SE, Gottlieb L, Toksvig-Larsen S, et al. Roentgen stereophotogrammetric analysis using computer-based image-analysis. $J$ Biomech 1997;30:993-5.

23. Schueler B, Hu X. Correction of image intensifier distortion for threedimensional x-ray angiography. In: Medical Imaging, Physics of Medical Imaging. San Diego, California, USA: SPIE 1995:272-9.

24. Kärrholm J, Selik G, Elmqvist LG, Hansson LI. Active knee motion after cruciate ligament rupture: stereoradiography. Acta Orthop Scand 1988;59:158-64. 